\title{
Cohesin occupancy and composition at enhancers and promoters are linked to DNA replication origin proximity in Drosophila
}

\author{
Michelle Pherson, ${ }^{1}$ Ziva Misulovin, ${ }^{1}$ Maria Gause, and Dale Dorsett \\ Edward A. Doisy Department of Biochemistry and Molecular Biology, Saint Louis University School of Medicine, Saint Louis, \\ Missouri 63104, USA
}

\begin{abstract}
Cohesin consists of the SMC1-SMC3-Rad21 tripartite ring and the SA protein that interacts with Rad21. The Nipped-B protein loads cohesin topologically around chromosomes to mediate sister chromatid cohesion and facilitate long-range control of gene transcription. It is largely unknown how Nipped-B and cohesin associate specifically with gene promoters and transcriptional enhancers, or how sister chromatid cohesion is established. Here, we use genome-wide chromatin immunoprecipitation in Drosophila cells to show that SA and the Fs(1)h (BRD4) BET domain protein help recruit Nipped-B and cohesin to enhancers and DNA replication origins, whereas the MED30 subunit of the Mediator complex directs Nipped-B and Vtd in Drosophila (also known as Rad21) to promoters. All enhancers and their neighboring promoters are close to DNA replication origins and bind SA with proportional levels of cohesin subunits. Most promoters are far from origins and lack SA but bind Nipped-B and Rad21 with subproportional amounts of SMCl, indicating that they bind cohesin rings only part of the time. Genetic data show that Nipped-B and Rad21 function together with Fs(1)h to facilitate Drosophila development. These findings show that Nipped-B and cohesin are differentially targeted to enhancers and promoters, and suggest models for how SA and DNA replication help establish sister chromatid cohesion and facilitate enhancer-promoter communication. They indicate that SA is not an obligatory cohesin subunit but a factor that controls cohesin location on chromosomes.
\end{abstract}

[Supplemental material is available for this article.]

Cohesin mediates sister chromatid cohesion to ensure accurate chromosome segregation and also plays roles in DNA repair and gene transcription (Dorsett and Ström 2012; Dorsett and Merkenschlager 2013; Uhlmann 2016; Morales and Losada 2018; Villa-Hernández and Bermejo 2018). In Drosophila, cohesin facilitates enhancer-promoter communication and regulates activity of the Polycomb repressive complex 1 at silenced and active genes (Rollins et al. 1999; Schaaf et al. 2013a,b; Pherson et al. 2017).

Cohesin structure and chromosome binding are relatively well understood. The SMC1, SMC3, and Rad21 subunits form a tripartite ring and SA interacts with Rad21. A Nipped-B-Mau2 complex loads cohesin topologically around chromosomes and a Pds5-Wapl complex removes cohesin. SA, Nipped-B, Pds5, and Wapl contain HEAT repeats and interact with cohesin to control its binding and activities (Neuwald and Hirano 2000; Wells et al. 2017). These accessory proteins facilitate ring opening to load and remove cohesin from chromosomes (Murayama and Uhlmann 2014, 2015; Çamdere et al. 2015; Beckouët et al. 2016; Elbatsh et al. 2016; Yu 2016; Ouyang and Yu 2017; Petela et al. 2018).

Less is known about how cohesin is targeted to sequences that control gene transcription or how sister chromatid cohesion is established. In Drosophila, cohesin associates with active genes, transcriptional enhancers, and the Polycomb response elements (PREs) that control epigenetic gene silencing (Misulovin et al. 2008, 2018; Schaaf et al. 2013a,b; Swain et al. 2016). Cohesin occupies all enhancers and PREs, and preferentially those active genes positioned

\footnotetext{
${ }^{1}$ These authors contributed equally to this work. Corresponding author: dale.dorsett@health.slu.edu

Article published online before print. Article, supplemental material, and publication date are at http://www.genome.org/cgi/doi/10.1101/gr.243832.118. Freely available online through the Genome Research Open Access option.
}

within several kilobases of the early DNA replication origins (MacAlpine et al. 2010; Misulovin et al. 2018).

The Pds5 and Wapl cohesin removal factors limit the size of cohesin domains surrounding early origins, whereas Pds5 and the Brca2 DNA repair protein, which form a complex lacking Wapl (Brough et al. 2012; Kusch 2015) have opposing effects on SA origin occupancy and sister chromatid cohesion (Misulovin et al. 2018). Pds5 is required for sister chromatid cohesion and facilitates SA binding, whereas Brca2 inhibits SA binding and counters the ability of Pds5 to support sister cohesion when Pds5 levels are low. These findings gave rise to the idea that Pds5 and SA function at replication origins to establish chromatid cohesion (Misulovin et al. 2018).

To gain more insight into how cohesin associates with gene regulatory sequences we used genome-wide chromatin immunoprecipitation sequencing (ChIP-seq) to investigate how multiple cohesin subunits occupy different genomic features in Drosophila cells. We also examined the roles of cohesin subunits, the Mediator complex, and the Fs(1)h (BRD4) BET domain protein in cohesin localization. The results indicate that cohesin associates with enhancers and most promoters by different mechanisms, and that proximity to DNA replication origins influences cohesin occupancy and composition.

\section{Results}

We compared how cohesin subunits and the Nipped-B cohesin loading factor (Fig. 1A) occupy promoters, enhancers, and

(c) 2019 Pherson et al. This article, published in Genome Research, is available under a Creative Commons License (Attribution 4.0 International), as described at http://creativecommons.org/licenses/by/4.0/. 


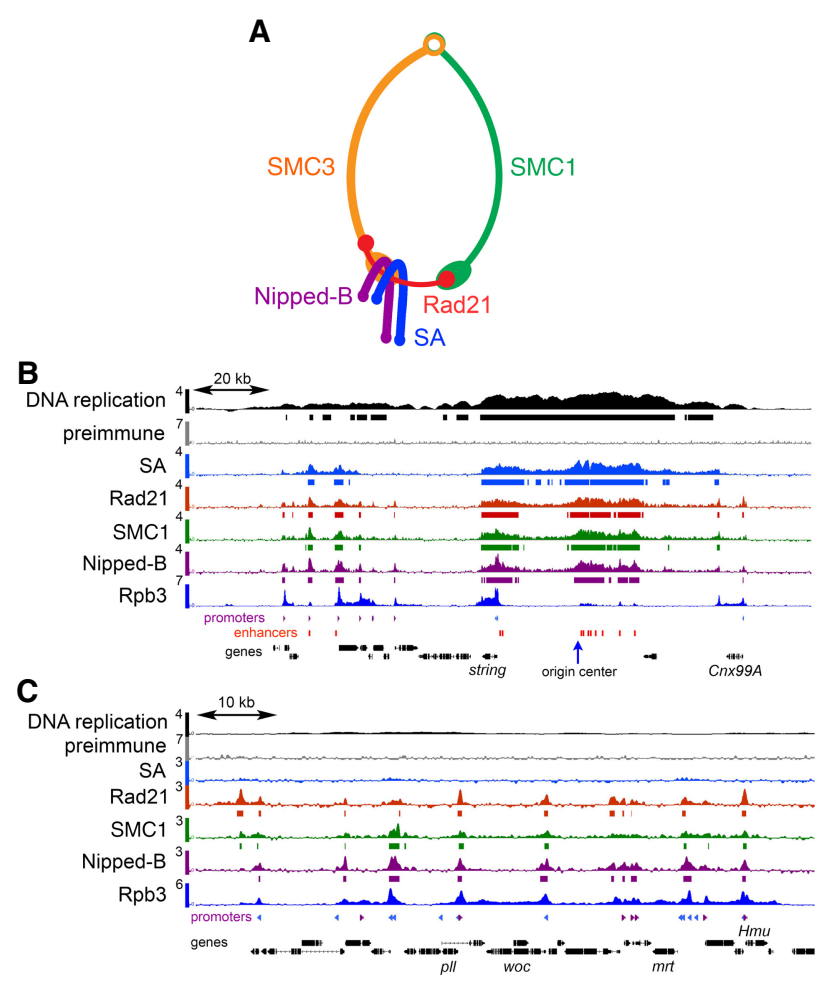

Figure 1. Cohesin and Nipped-B ChIP-seg in BG3 cells. (A) Cohesin subunit structure. $(B)$ ChIP-seq near an early DNA replication origin at the string (stg, also known as cdc25) gene. ChIP-seq and DNA replication data are plotted as $\log _{2}$ enrichment. Bars below each track indicate enrichment in the 95th percentile over regions $\geq 300 \mathrm{bp}$. Rpll33 (also known as Rpb3) RNA polymerase II subunit data are from a prior publication (Pherson et al. 2017). Locations of promoters (purple and blue, forward and reverse) and enhancers (red) are indicated below the tracks. (C) ChIP-seq in a region distant from an early replication origin containing the woc (without children) gene. quences (RAN). SA does not occupy most of the 7389 active promoters, but essentially all 2353 enhancers, 195 PREs, and 78 origin centers. In contrast, $\operatorname{Rad} 21, \mathrm{SMC} 1$, and Nipped-B occupy most active promoters and all enhancers, PREs, and origins. SA has the highest median occupancy at origins, whereas the Rad21 and SMC1 ring components are highest at enhancers and Nipped-B is maximal at PREs (Fig. 2A; Supplemental Table S2).

Differential occupancy by cohesin subunits is also illustrated by meta-analyses that average the distribution of ChIP-seq enrichment centered at each type of feature (Fig. 2B). Of the four proteins, Nipped-B (purple) shows the highest mean enrichment at promoters, enhancers, PREs, and the second highest at origins (Fig. 2B). SA is the highest at origins. At promoters, there is minimal SA (blue), and Rad21 enrichment (red) is higher than SMC1 (green). Rad21 enrichment is also higher than SMC1 at origin centers. In contrast, SMC1 shows higher enrichment than Rad21 at enhancers and PREs. At enhancers, SA extends into the flanking regions more than Rad21 and SMC1, suggesting that some SA binds independently of cohesin (Fig. 2B).

Enrichment values for the individual cohesin subunits depend on different efficiencies of crosslinking and precipitation and thus cannot be directly compared. However, we infer that the stoichiometry of the subunits varies between the different features because their relative mean enrichments differ. As described below, depletion experiments confirm that epitope masking or differential crosslinking are not responsible for low SA and SMC1 levels seen at most promoters.

SA shows higher mean enrichment relative to the other cohesin subunits in meta-origin analysis but lower enrichment at promoters and enhancers (Fig. 2B). This indicates that cohesin at features close to origins has more SA than cohesin at origin-distal features and/or that some SA binds near origins independently of cohesin. As described below, we find that enhancers and the rare promoters that bind SA are origin-proximal and that promoters with low SA are origin-distal.
Polycomb Response Elements (PREs) by ChIP-seq in ML-DmBG3-c2 (BG3) cells derived from third instar central nervous system. Multiple biological replicates were used for each protein (Supplemental Table S1). Figure 1B shows ChIP-seq in a region near an early DNA replication origin where cohesin levels are high, and Figure 1C shows an origin-distal region with lower occupancy. Preimmune serum ChIP-seq shows insignificant enrichment of functional features (Fig. 1; Supplemental Fig. S1).

\section{Cohesin subunits occupy functional features in different proportions}

Figure 2A illustrates the distributions of the SA, Vtd (also known as Rad21), SMC1, and Nipped-B occupancies of promoters (PRO), enhancers (ENH), PREs (PRE), and centers of early DNA replication origins (ORI) using violin plots. All show insignificant median enrichment across 6892 randomly positioned se-
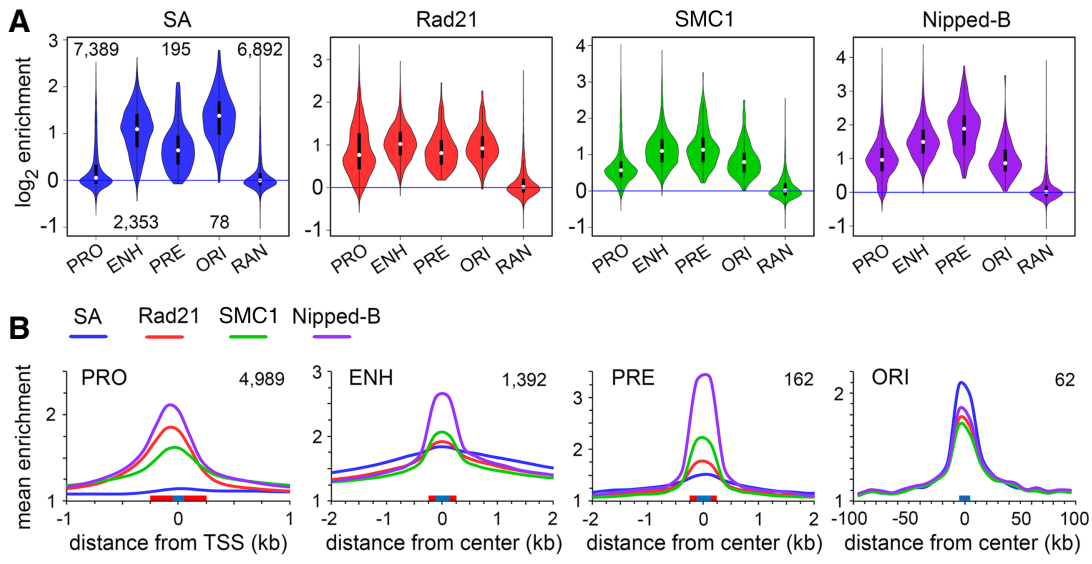

Figure 2. Cohesin subunits are present in different ratios at promoters, enhancers, PREs, and replication origins in BG3 cells. (A) Violin plot distributions of SA (blue), Rad21 (red), SMC1 (green), and Nipped-B (purple) at active promoters (PRO), enhancers (ENH), Polycomb Response Elements (PRE), early replication origin centers (ORI), and randomly positioned sequences (RAN). The numbers of each type of feature analyzed are indicated in the SA plot. White dots are the median values given in Supplemental Table S2. (B) Meta-analyses of promoters, enhancers, PREs, and early replication origins for SA (blue), Rad21 (red), SMC1 (green), and Nipped-B (purple). Red boxes on the $x$-axes indicate the feature sizes used to calculate occupancy for the violin plots, and blue boxes indicate the bin sizes used to average enrichment for the meta-analysis. The numbers of each type of feature used for meta-analysis are indicated in the upper right corner of each graph. These are less than for the violin plots because features that overlap in the meta-analysis region were removed to minimize distortions. 


\section{Nipped-B and Rad21 occupy most gene promoters without SA}

Figure 1C shows origin-distal promoters occupied by Nipped-B, Rad21, and SMC1 with little or no SA. Dot plots of Rad21 promoter occupancy against SA (Fig. 3A) or SMC1 (Fig. 3B) show that most promoters have Rad21, subproportional SMC1, and no SA (black arrows). We defined "high SA" promoters as those within regions of SA enrichment in the 95th percentile. These regions, ranging from 300 bp to several kilobases, are marked by bars below the SA ChIP-seq track in Figure 1B. High SA promoters represent $12 \%$ of active promoters $(895 / 7398)$ and are plotted in red in the dot plots (Fig. 3A,B). In contrast to most promoters, high SA promoters have proportional SA, Rad21, and SMC1 levels similar to enhancers and PREs (Fig. 3A,B). This implies that cohesin subunits are more stoichiometric at high SA promoters and enhancers than at most promoters.

Subproportional SMC1 levels at SA-deficient promoters implies that Nipped-B and Rad21 occupy these promoters in both the absence and presence of SMC1-SMC3 dimers. We envision that a percentage of each of these promoters in a cell population bind Nipped-B and Rad21 without SMC1 and SMC3, while another fraction has SMC1-SMC3-Rad21 rings. In contrast, enhancers and high SA promoters are primarily occupied by complete cohesin complexes.

Meta-analysis using Rpb3 ChIP-seq data (Pherson et al. 2017) shows that high SA promoters (red) have more RNA polymerase on average than most promoters (blue) (Fig. 3C). Rpb3 peaks downstream from the transcription start site (TSS) at $+30 \mathrm{bp}$ for high SA promoters and at +65 for all promoters (red and blue arrowheads). This agrees with PRO-seq studies showing that genes with more cohesin show above average transcription and promoter-proximal Pol II pausing (Schaaf et al. 2013a).

The positions of different cohesin subunits relative to the transcription start site differ at promoters. Averaging all promoters, Nipped-B and Rad21 peak 70 bp upstream of the start site ( $-70 \mathrm{bp}$, purple and red arrowheads), whereas SMC1 peaks at -35 bp (green arrowhead) (Fig. 3D). We interpret this as indicating that some Rad21 binds to promoters independently and upstream of SMC1, and another fraction interacts with SMC1 downstream.

Cohesin is positioned further downstream at high SA promoters compared to most promoters (Fig. 3D). Nipped-B peaks upstream at -50 bp (purple arrowhead), but Rad21 (red) and SMC1 (green) peak together just downstream from the transcription start site, and SA (blue) peaks further downstream near +100 (Fig. 3D). SA also extends more than Rad21 and SMC1 into the transcribed region. Pol II (Rpb3) peaks 30 bp downstream from Rad21 and SMC1 but nearly $70 \mathrm{bp}$ upstream of SA. We theorize that some SA associates with the elongating Pol II complex that enters into the gene body. The precise alignment of Rad21 and SMC1 peaks at high SA promoters contrasts with their misalignment at most promoters and correlates with their more proportional levels. We interpret this as indicating that high SA promoters are occupied primarily by complete cohesin complexes.

High-SA promoters and enhancers are close to early DNA replication origins

As noted above, SA levels are highest near early replication origins, suggesting that high SA promoters are positioned close to origins and those with low SA are located farther away. We tested this by comparing the levels of early $S$ phase DNA synthesis at functional features. Those close to early origins should be replicated in early S phase. We measured early DNA synthesis by incorporation of the EdU thymidine analog and highthroughput sequencing in cells blocked in early S phase with hydroxyurea. The results are similar to data obtained using BrdU and microarrays (Eaton et al. 2011). As predicted, high SA promoters and enhancers experience higher DNA replication in early $S$ phase than most promoters (Fig. 3E). PREs and randomly positioned sequences show low replication and origin centers show high levels, as expected.

\section{Genome Research}

www.genome.org 
Extragenic enhancers located outside of transcribed regions experience higher early $\mathrm{S}$ phase DNA synthesis than most enhancers, indicating that they are positioned particularly close to early origins (Fig. 3E). The origin recognition complex (ORC) recruits the MCM2-7 helicase complex that unwinds duplex DNA to start replication during origin licensing in early G1 and transcription pushes MCM2-7 to regions outside of genes (Powell et al. 2015). Repositioning of MCM2-7 outside of transcribed regions can explain higher early DNA synthesis at extragenic enhancers and raises the possibility that enhancers may help position MCM2-7. Supplemental Figure S2A illustrates the overlap of early origins with clusters of enhancers.

\section{SA facilitates cohesin and Nipped-B occupancy of enhancers and origin- proximal promoters}

We depleted SA by RNAi in BG3 cells and conducted ChIP-seq for Nipped-B, Rad21, and SMC1 to test if SA positions cohesin at origin-proximal features. RNAi was conducted for 3-4 d, depleting SA by $80 \%-90 \%$ without reducing sister chromatid cohesion and slightly slowing cell proliferation (Supplemental Fig. S3; Schaaf et al. 2009; Misulovin et al. 2018). SA depletion partially reduces total Rad21 protein and does not alter total Nipped-B levels (Supplemental Fig. S3; Schaaf et al. 2009). BG3 cells divide during the depletion with a 24-h cycle, and changes revealed by ChIP-seq reflect an altered steady-state equilibrium of cohesin occupancy.

Figure 4 shows that SA depletion shifts cohesin occupancy from origin-proximal to origin-distal features, supporting the idea that SA positions cohesin close to origins. Rad21 (Fig. 4A), SMC1 (Fig. 4B), and Nipped-B (Fig. 4C) increase at most promoters upon SA depletion and decrease at enhancers and origin-proximal regions. They also decrease at PREs with the exception that SMC1 PRE occupancy is only slightly modified. The changes of cohesin occupancy are statistically significant except for SMC1 at PREs (Supplemental Table S2). Supplemental Figure S2B illustrates how Rad21, SMC1, and Nipped-B decrease at the string enhancers and flanking promoters near a replication origin. In contrast, Rad21 and SMC1 increase slightly at some origin-distal promoters in Supplemental Figure S2C. Promoter dot plots in Figure 4 show that although Rad21, SMC1, and Nipped-B decrease at the origin-proximal high SA promoters (red dots), they increase at low SA origin-distal promoters (blue dots).

SA depletion reduces total Rad21 protein levels (Supplemental Fig. S3), and part of the Rad21 decrease at enhancers and origin-proximal promoters may reflect reduced protein levels. Against this idea, Rad21 increases at the origin-distal promoters to levels that are higher than those left on the enhancers, indicating that the remaining amount of total Rad21 does not limit chromosome association. Also, total Nipped-B protein levels are not reduced by SA depletion and Nipped-B also shows a decrease at origin-proximal features and increase at origin-distal promoters. FRAP experiments show that $<20 \%$ of cohesin in the nucleus is bound to chromosomes in Drosophila cells, indicating that total cohesin levels are not limiting for chromosome association (Gause et al. 2010).

The opposite effects of SA depletion on Rad21, SMC1, and Nipped-B association with origin-proximal features and origin-distal promoters indicates that epitope masking or poor crosslinking are not responsible for the inability to detect SA at origin-distal promoters. Rad21, SMC1, and Nipped-B all decrease at originproximal promoters and enhancers where SA is detected but increase at origin-distal promoters where SA is not detected. If SA were present at origin-distal promoters, we would expect Rad21, SMC1, and Nipped-B levels to also decrease upon SA depletion. The Rad21, SMC1, and Nipped-B increases at origin-distal promoters with SA depletion also provide further evidence that cohesin can bind promoters independently of SA.

SA is not part of the cohesin ring (Fig. 1A) and is not required for cohesin to topologically bind chromosomes (Kulemzina et al. 2012). We depleted SMC1 to compare how a ring component influences Nipped-B and cohesin subunit chromosome occupancy (Fig. 5). The SMC1 antibody gives weak signals in western blots so ChIP-seq was used to confirm SMC1 depletion, showing that SMC1 occupancy is globally reduced (Fig. 5A; Supplemental Table S2). Supplemental Figure S4 shows examples of SMC1 reduction at 
A

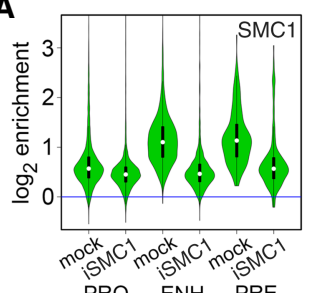

B

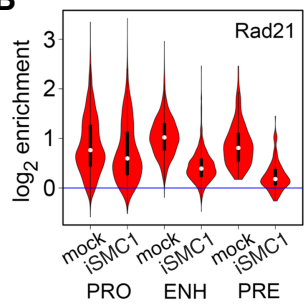

C

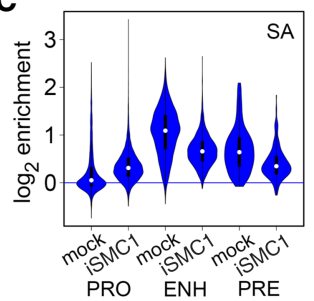

D

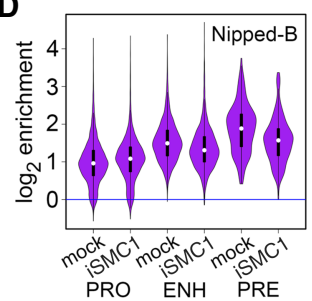

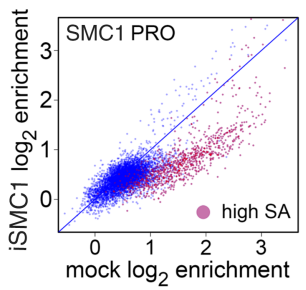
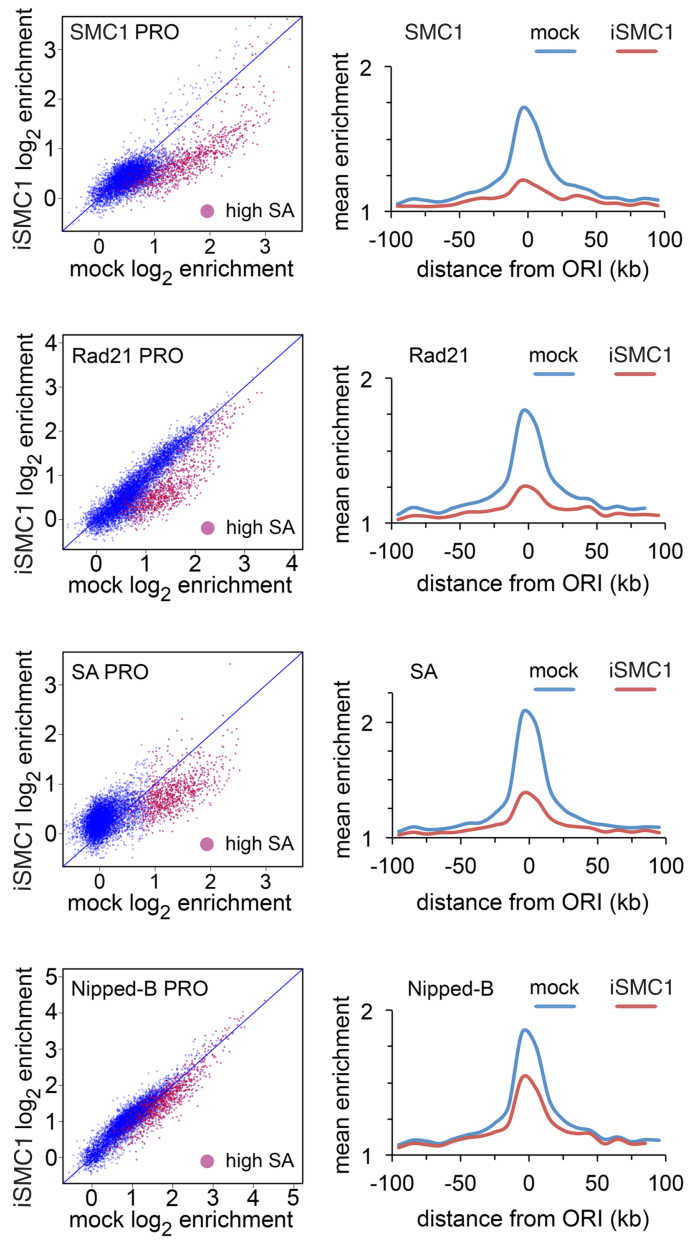

Figure 5. $S M C 1$ facilitates Rad21 and $S A$ association with enhancers and origin-proximal promoters in BG3 cells. (A) SMC1 ChIP-seq of SMC1-depleted (iSMC1) cells. The violin plot distributions, promoter dot plots, and meta-analysis of early DNA replication origins show globally reduced SMC1 occupancy leaving similar residual levels at all features. (B) Effects of SMC1 depletion on Rad21 localization. (C) Effects of SMC1 depletion on SA localization. (D) Effects of SMC1 depletion on Nipped-B localization. All occupancy changes are statistically significant (Supplemental Table S2). ChIP-seq track examples are in Supplemental Figure S4.

the origin-proximal region containing the string gene and an origin-distal region containing woc. SMC1 depletion reduces total Rad21 protein to a similar extent as SA depletion (Supplemental Fig. S3). SMC1 depletion also reduces total Rad21 in human cells (Laugsch et al. 2013).

SMC1 depletion reduces Rad21 at enhancers, PREs, and originproximal promoters (red dots in promoter dot plots) (Fig. 5B; Supplemental Table S2; Supplemental Fig. S4A). However, as illustrated by promoter dot plot in Figure 5B (blue dots) and ChIP-seq tracks in Supplemental Figure S4B, SMC1 depletion has little effect on Rad21 at origin-distal promoters, and the overall Rad21 level at promoters is now higher than at enhancers (violin plot). Some of the Rad21 decrease at the origin-proximal features might reflect reduced total Rad21 protein, but the minimal effect at origin-distal promoters indicates that the total Rad21 level does not limit chromosome association. The finding that SMC1 depletion reduces Rad21 levels at origin-proximal promoters, where SMC1 is detected, but has little effect at origin-distal promoters indicates that epitope masking or poor crosslinking are not responsible for the subpropor- tional SMC1 at origin-distal promoters seen in control cells. These findings also confirm that Rad 21 can occupy promoters independently of SMC1 and SA.

SA association with enhancers, PREs, and origin-proximal regions is also SMC1-dependent (Fig. 5C; Supplemental Table S2; Supplemental Fig. S4A). SMC1 depletion decreases SA at high SA promoters and increases SA to modest levels at promoters that normally have no SA (Fig. 5C; Supplemental Fig. S4B). Detection of SA at origin-distal promoters with SMC1 depletion indicates that epitope masking or poor crosslinking does not cause the lack of SA at these promoters in control cells.

Nipped-B associates with promoters independently of SMC1. SMC1 depletion causes statistically significant reductions in Nipped-B at enhancers, PREs, and origin-proximal promoters with slight increases at origin-distal promoters (Fig. 5D; Supplemental Table S2; Supplemental Fig. S4).

Supplemental Figure S5 shows that Rad21 depletion reduces Nipped-B association with origin-proximal features, including enhancers and high SA promoters, and increases Nipped-B at most promoters. All changes are statistically significant (Supplemental Table S2). We conclude that Nipped-B enhancer occupancy depends on SA and Rad21, whereas association with most promoters is cohesin-independent.

\section{The MED30 Mediator subunit and the Fs(1)h BET domain protein colocalize with Nipped-B and cohesin}

The preceding experiments show that SA promotes Nipped-B and cohesin occupancy of features close to early origins and that Nipped-B and Rad21 bind origin-distal promoters independently of SA and SMC1. This implies that enhancer and promoter factors differentially recruit Nipped-B, SA, and cohesin. The Mediator complex that regulates transcription (Allen and Taatjes 2015) and the BRD4 BET domain protein that binds acetylated histones (Hsu and Blobel 2017) are candidates for such factors. Mammalian Mediator interacts with NIPBL (Nipped-B) (Kagey et al. 2010) and an affinity chromatography-mass spectrometry screen revealed that the Drosophila MED30 Mediator subunit interacts with Nipped-B (Guruharsha et al. 2012). It was recently reported that human BRD4 interacts with NIPBL and that BRD4 mutations cause birth defects similar to those caused by NIPBL mutations (Olley et al. 2018).

We performed ChIP-seq for MED1, MED30, and Fs(1)h, the Drosophila ortholog of BRD4, to compare them to cohesin. Figure $6 \mathrm{~A}$ shows the origin-proximal string locus, and Figure $6 \mathrm{~B}$ shows an origin-distal region. MED30 and Fs(1)h spread similarly to Nipped-B at the string enhancers, and MED1 displays more distinct

\section{Genome Research}

www.genome.org 
A

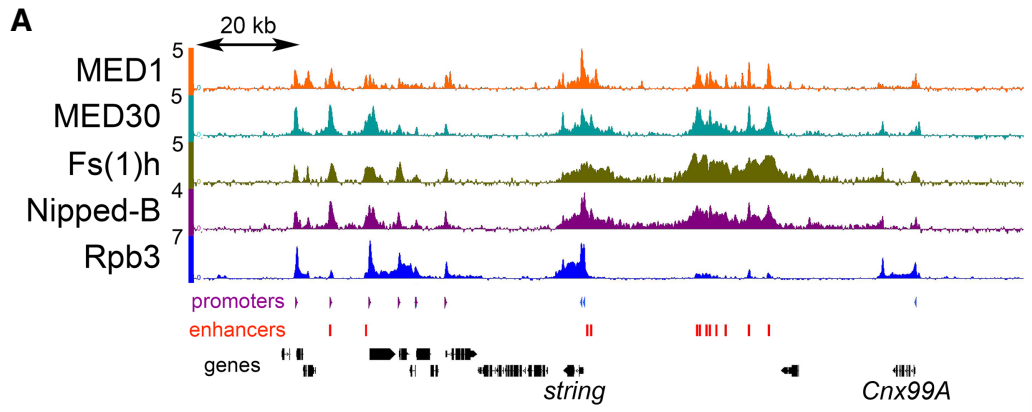

B

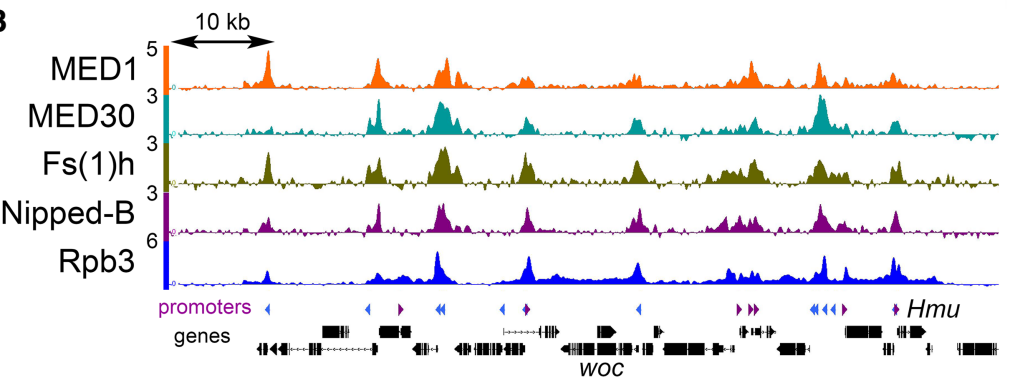

C

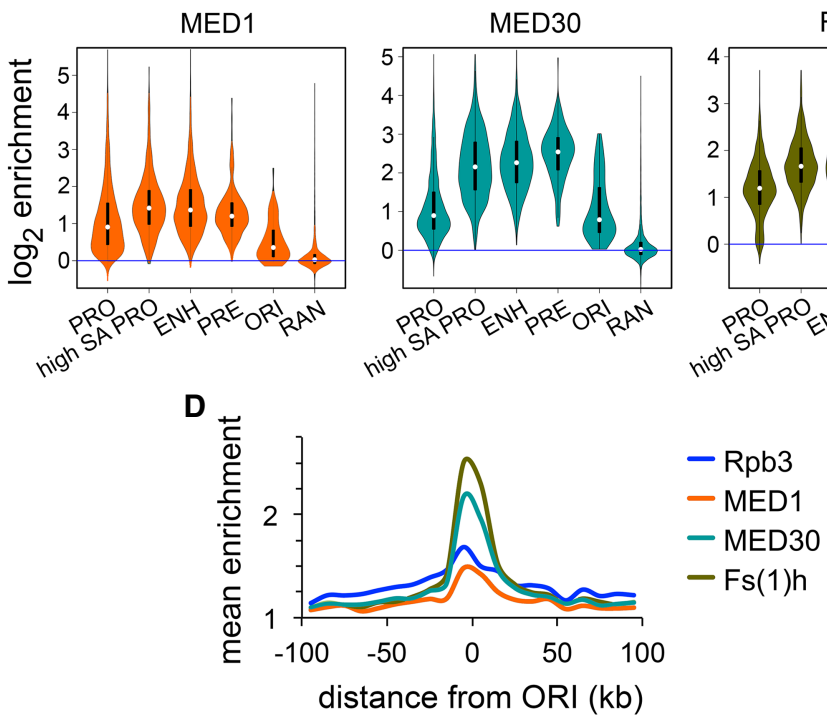

Figure 6. The MED30 Mediator subunit and Fs(1)h BET domain protein colocalize with Nipped-B and are origin-centric in BG3 cells. $(A)$ ChIP-seq for the indicated proteins in an origin-proximal region containing the string $(c d c 25)$ gene. $(B)$ ChIP-seq for the indicated proteins in an origin-distal region containing the woc gene. (C) Violin plot distributions for MED1 (orange), MED30 (cyan), and $\mathrm{Fs}$ (1)h (olive) occupancy at all active promoters (PRO), SA-occupied promoters (high SA PRO), enhancers (ENH), PREs (PRE), centers of early DNA replication origins (ORI), and randomly positioned sequences (RAN). (D) Meta-origin analysis of Rpb3 (blue), MED1 (orange), MED30 (cyan), and Fs(1)h (olive) occupancy.

peaks (Fig. 6A). MED30 and Fs(1)h are high at enhancers (Fig. 6C) and strongly origin-centric (Fig 6D). MED1 is less origin-centric, similar to RNA polymerase (Rpb3). The origin-centric distribution of MED30 and Fs(1)h led us to test how they influence Nipped-B association with enhancers and promoters.

\section{MED30 facilitates Nipped-B association with promoters, and Fs(1)h promotes Nipped-B enhancer occupancy}

MED30 depletion decreases Nipped-B at all promoters with slight increases at enhancers and PREs (Fig. 7A; Supplemental
Table S2). Thus, although MED30 is at both promoters and enhancers, it facilitates Nipped-B association only at promoters, suggesting that other factors influence the ability of MED30 to recruit Nipped-B.

MED30 depletion increases SA at all features (Fig. 7B; Supplemental Table S2), and SA depletion globally increases MED30 occupancy (Supplemental Fig. S6A; Supplemental Table S2), suggesting that SA and MED30 compete for binding. SMC1 depletion slightly reduces MED30 at all features (Supplemental Fig. S6B), indicating that the SA-MED30 competition is specific.

Fs(1)h promotes Nipped-B association with enhancers and origin-proximal promoters (Fig. 8A; Supplemental Table S2). We treated cells with the JQ1 inhibitor of BET domain binding to acetylated histones (Filippakopoulos et al. 2010) for $8 \mathrm{~h}$ to globally reduce Fs(1)h binding (Supplemental Fig. S7A). JQ1 treatment reduces Nipped-B at enhancers, high SA promoters, and PREs with an overall decrease in origin-proximal regions. There is little effect at origin-distal promoters (Fig. 8A).

The effects of JQ1 treatment on Rad21 are similar to the effects on Nipped-B, with decreases at enhancers, high SA origin-proximal promoters, and PREs and little effect at origin-distal promoters (Fig. 8B; Supplemental Table S2). SMC1 slightly increases at origin-distal promoters and decreases at enhancers, with little change at high SA promoters and increases at many PREs (Fig. 8C; Supplemental Table S2). JQ1 slightly reduces SA at high SA promoters, enhancers, and PREs, with little effect at most promoters (Fig. 8D; Supplemental Table S2). JQ1 modestly reduces MED30 at enhancers, with even smaller effects at other features (Supplemental Table S2; Supplemental Fig. S6C).

The picture that emerges is that Fs(1)h facilitates Nipped-B and Rad21 association with enhancers, but only slightly influences SA and SMC1 occupancy. This suggests that SA and SMC1 can be recruited independently of Nipped-B and Rad21 to enhancers. JQ1 stops most cells in the G2 phase of the cell cycle (Supplemental Fig. S7B). Thus, some effects on cohesin distribution could reflect differences between populations in which roughly half the cells are in G2 (control) as opposed to the majority (JQ1). This might explain minor changes in SA and SMC1, but seems unlikely to cause the significant Nipped-B and Rad21 decreases at enhancers. Indeed, as described below, Nipped-B, vtd (Rad21), and $f s(1) h \mathrm{mu}-$ tations show genetic interactions during development when cell division is not blocked. 

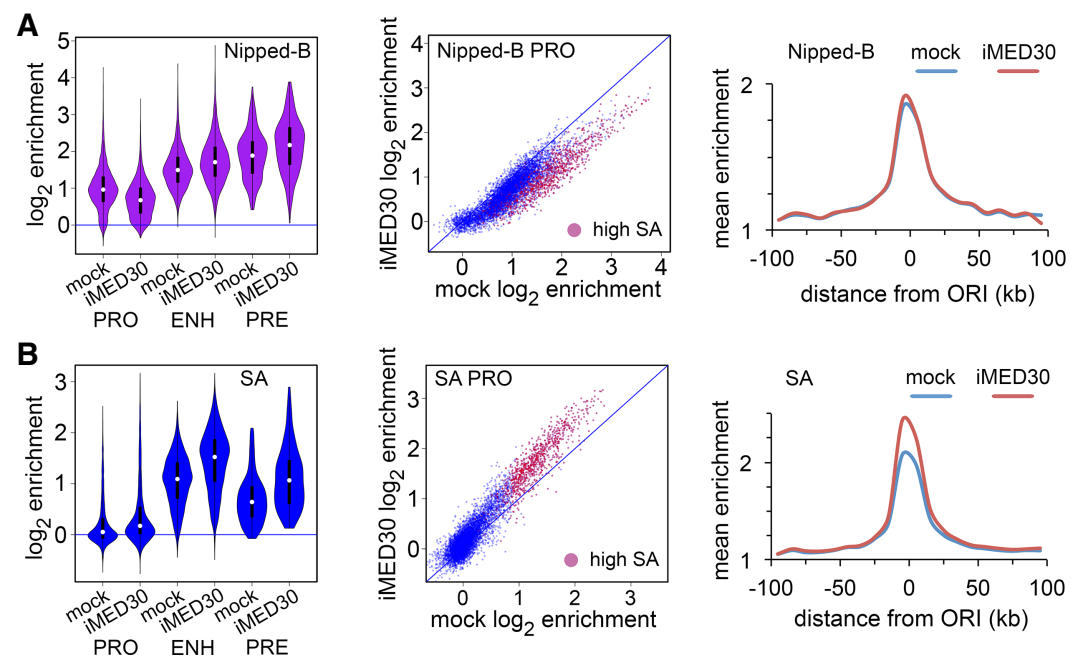

Figure 7. The MED30 Mediator subunit facilitates Nipped-B association with promoters in BG3 cells. An example of MED30 protein depletion is in Supplemental Figure S3A. (A) Effects of MED30 depletion (iMED30) on Nipped-B localization. (B) Effects of MED30 depletion on SA localization. All changes in Nipped-B and SA occupancy are statistically significant except at the centers of early origins (Supplemental Table S2).

\section{Nipped-B and Rad21 (vtd) mutations interact genetically with the $f_{s}(1) h^{l}$ hypomorphic mutation}

We tested the in vivo significance of Fs(1)h effects on Nipped-B and Rad21 localization using the hypomorphic $f s(1) h^{1}$ mutation. $f s(1) h^{1}$ was recovered in a screen for female-sterile mutations on the X Chromosome (Gans et al. 1975). Null $f_{s}(1) h$ alleles are lethal, but $f s(1) h^{1}$ is a viable missense mutation (Digan et al. 1986; Florence and Faller 2008).

Nipped-B and Rad21 (vtd) mutations dominantly reduce $f s(1) h^{1}$ viability (Fig. 9A). At $29^{\circ}, 62 \%$ of the expected $f s(1) h^{1} / Y$ males were recovered relative to their $f s(1) h^{1} /+$ sisters. The heterozygous Nipped- $B^{407}$ null mutation does not slow development or reduce viability in multiple wild-type backgrounds (Rollins et al. 1999; Wu et al. 2015) but reduces viability of $f s(1) h^{1} / Y$ males to $5 \%$ of their $f s(1) h^{1} /+$; Nipped-B $B^{407} /+$ sisters. Nipped- $B^{N C 41}$, a truncation mutation (Gause et al. 2008) dominantly reduces $f s(1) h^{1}$ male viability to $25 \%$. $v t d^{4}$, a partial Rad21 deletion (Hallson et al. 2008) dominantly reduces $f s(1) h^{1}$ male viability to $12 \%$, and $v t d^{\text {ex } 15}$, another partial deletion (Pauli et al. 2008) reduces viability to $49 \%$. All viability reductions are statistically significant, except with $v t d^{\text {ex15. }}$.

Nipped-B and Rad21 (vtd) mutations dominantly suppress homeotic transformations caused by Pc mutations (Kennison and Tamkun 1988; Hallson et al. 2008; Schaaf et al. 2013b). fs(1) $h^{1}$ similarly suppresses ectopic sex comb bristles on the T2 and T3 legs of $P c^{4} /+$ males (Fig. 9B). Combining $f s(1) h^{1}$ with heterozygous Nipped- $B^{407}$ reduces the number of T1 sex comb bristles in the surviving males (Fig. 9B). The genetic interactions between $f s(1) h$, Nipped-B, and Rad21 indicate that the proteins they encode function together in vivo.

\section{Discussion}

Our experiments show that SA helps recruit complete cohesin complexes to enhancers, which are all located close to early DNA replication origins (Fig. 10A) and to those promoters that are also close to origins. Nipped-B and Rad 21 also occupy origin-distal promoters, which bind cohesin rings only part of the time (Fig. 10A).
The MED30 subunit of the Mediator complex facilitates association of Nipped-B and Rad21 with all promoters and the $\mathrm{Fs}(1) \mathrm{h}$ (BRD4) mitotic bookmarking protein facilitates cohesin association with enhancers and the origin-proximal promoters (Fig. 10A). Genetic evidence shows that Fs(1)h functions together with Nipped-B and Rad21 in vivo to support development.

\section{Enhancer-promoter communication}

Only those promoters that are close to enhancers and origins are occupied primarily by complete cohesin complexes. We thus theorize that these are the promoters that are targeted by enhancers. We envision that DNA replication pushes cohesin from enhancers to origin-proximal promoters (Fig. 10B) based on the evidence that replication origins form preferentially at enhancers and prior indications that replication pushes cohesin (Kanke et al. 2016; Misulovin et al. 2018). We do not know if the Nipped-B and Rad21 that bind origin-distal promoters independently of SA and SMC1 (Fig. 10A) influence gene transcription. This will be challenging to unravel because Nipped-B and Rad21 are essential for complete cohesin rings to bind to chromosomes.

Since it was discovered that sister chromatid cohesion proteins facilitate expression of enhancer-activated genes (Rollins et al. 1999), it has been proposed that enhancer-promoter looping could be supported by intra-chromosomal cohesion. In the simplest version, a cohesin ring topologically encircles DNA near both the enhancer and the promoter to hold them together. The cohesin at the enhancer and promoter are thus the same molecules. Some of our findings argue against this idea. In particular, MED30 depletion reduces Nipped-B and Rad21 at origin-proximal promoters but not at the linked enhancers, indicating that different cohesin molecules are present at the enhancers and promoters. It could be that a cohesin ring at a promoter interacts with another at an enhancer to handcuff them together, or that cohesin interacts with Mediator, BRD4, or other proteins to stabilize enhancer-promoter looping.

Cohesin is removed from chromosomes at mitosis and loaded in early G1. Thus, the idea that DNA replication localizes cohesin to facilitate enhancer-promoter communication raises the question of how cohesin supports enhancer function in G1 before replication. One idea is that mitotic bookmarking factors facilitate cohesin loading at enhancers and target promoters. The BRD4 ortholog of $\mathrm{Fs}(1) \mathrm{h}$ remains bound to mitotic chromosomes and promotes rapid reactivation of transcription after cell division (Dey et al. 2000; Zhao et al. 2011). Thus, the finding that inhibiting Fs(1)h chromosome binding reduces Nipped-B and Rad21 at enhancers and origin-proximal promoters without going through cell division supports the idea that Fs(1)h marks them for cohesin loading.

\section{Sister chromatid cohesion}

We hypothesize that origins form at enhancers because enhancers trap the sliding MCM2-7 helicase that will initiate DNA replication

\section{Genome Research}

www.genome.org 

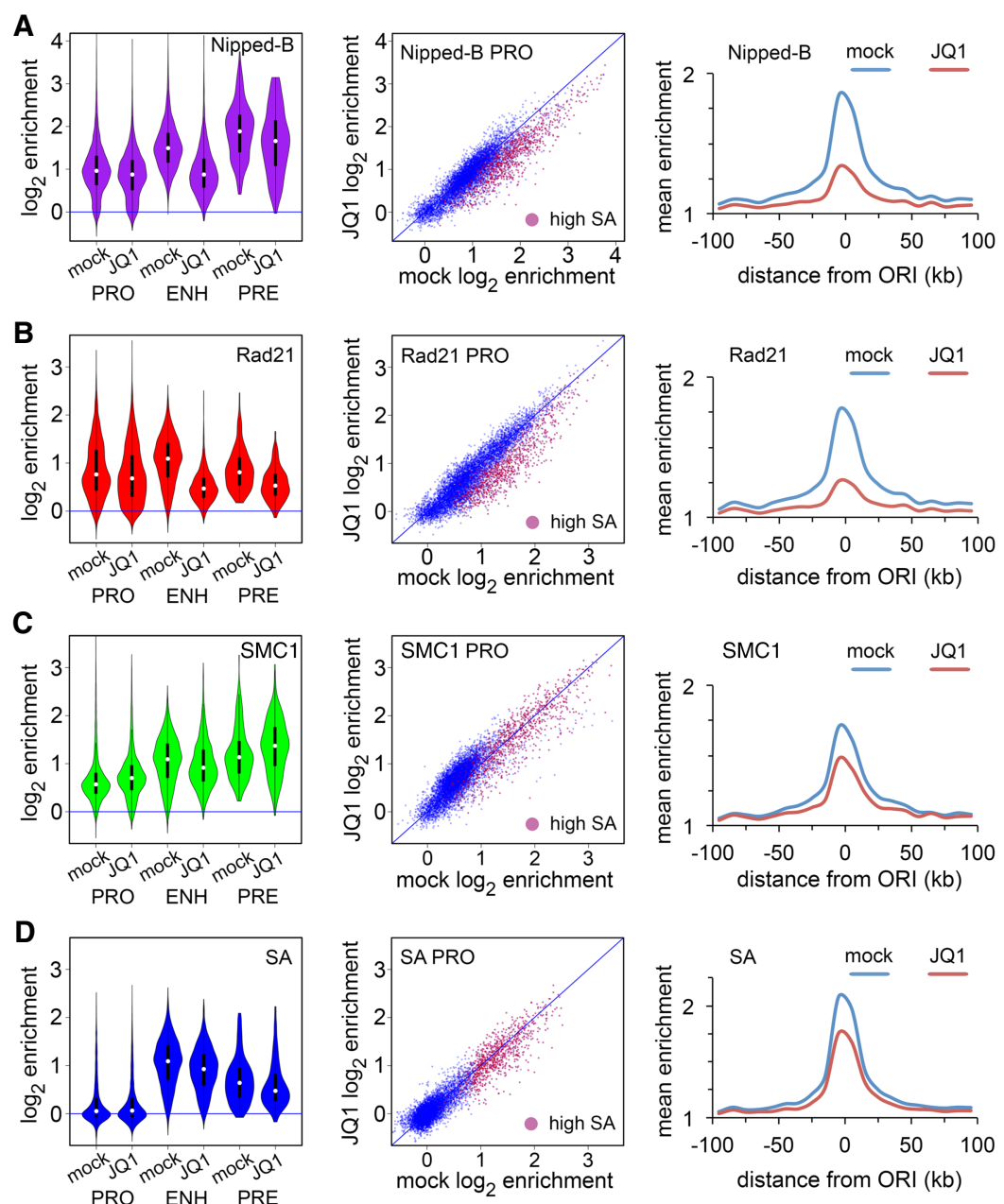

Figure 8. The $\mathrm{Fs}_{\mathrm{s}}(1) \mathrm{h} \mathrm{BET}$ domain protein promotes association of Nipped-B and $\operatorname{Rad} 21$ with enhancers and origin-proximal promoters. The effects of the JQ1 inhibitor on $\mathrm{Fs}(1) \mathrm{h}$ binding and cell cycle are shown in Supplemental Figure S6. (A) Effects of JQ1 on Nipped-B occupancy. (B) Effects of JQ1 on Rad21 occupancy. (C) Effects of JQ1 on SMC1 occupancy. (D) Effects of JQ1 on SA occupancy. Effects of JQ1 on Nipped-B occupancy are statistically significant except at PREs (Supplemental Table S2). All effects on Rad21 occupancy are significant. Effects on SMC1 occupancy are significant except at high SA promoters. Changes in SA occupancy are significant except at promoters and PREs.

(Fig. 10B). Localization of cohesin to enhancers and origins suggests a simple model for how sister chromatid cohesion is established. Upon initial unwinding of the DNA template by MCM2-7, cohesin behind the nascent replication forks encircles the two single-stranded templates, passively establishing cohesion while cohesin in front of the forks is pushed to origin-proximal promoters (Fig. 10B).

This model explains why Pds5, a cohesin removal factor, and SA, which is not required for cohesin to bind chromosomes topologically (Kulemzina et al. 2012), are required for sister chromatid cohesion. By positioning cohesin at enhancers, they ensure that the nascent sister chromatids will be topologically trapped within cohesin (Fig. 10B). This does not require that replisomes move through cohesin or new cohesin loading behind the fork as proposed in other models (Uhlmann 2016; Villa-Hernández and Bermejo 2018). It is consistent with the finding that cohesin can remain chromosome-bound and establish cohesion during DNA replication in the absence of the Wapl removal factor (Rhodes et al. 2017).
Parallels with vertebrate cohesin

Mammals have two SA orthologs, SA1 (STAG1) and SA2 (STAG2). Only SA2-containing cohesin is present at enhancers in human cells (Kojic et al. 2018), suggesting that SA2 is the functional ortholog of Drosophila SA. SA2 binds DNA independently of cohesin in vitro with a preference for single-stranded DNA and structures resembling replication forks (Countryman et al. 2018). This is consistent with our findings that $\mathrm{SA}$ is origin-centric and spreads further than cohesin around enhancers.

Mutations in the STAG2 gene encoding SA2 cause intellectual and growth deficits overlapping those seen in cohesinopathies caused by mutations in NIPBL or cohesin subunit genes (Mullegama et al. 2017, 2019; Soardi et al. 2017; Yuan et al. 2018). Individuals with $B R D 4$ mutations display similar birth defects, and BRD4 and NIPBL colocalize at enhancers (Olley et al. 2018). These studies agree with our findings that SA and $\mathrm{Fs}(1) \mathrm{h}$ facilitate association of Nipped-B and $\operatorname{Rad} 21$ with enhancers and that $\mathrm{Fs}(1) \mathrm{h}$ and Nipped-B function together in development.

Our data show parallels with cohesin loading in Xenopus. Cohesin loading in Xenopus oocyte extracts requires assembly of the prereplication complex that licenses replication origins and the Cdc7-Drf1 kinase that activates the prereplication complex interacts with NIPBL (Gillespie and Hirano 2004; Takahashi et al. 2004, 2008). This places cohesin at the site of replication initiation, similar to the role of SA in Drosophila.

Specialized DNA replication factors are needed to establish sister chromatid cohesion in yeast (Skibbens 2009), but it is unclear whether they are required at progressing forks or only upon initiation of replication. A study in human cells showed that NIPBL and cohesin interact with the MCM2-7 helicase (Zheng et al. 2018). The authors suggest that NIPBL bound to MCM2-7 is transiently held by the replisome and transferred behind the fork to load cohesin and establish sister cohesion, but it is possible that interactions with NIPBL could also trap MCM2-7 at enhancers prior to replication. Whether or not recruiting both MCM2-7 and cohesin to origins is sufficient to establish cohesion or whether cohesion requires new cohesin loading behind the replication fork remains to be resolved.

\section{Methods}

\section{Cell culture, RNAi depletion, and JQ1 treatment}

ML-DmBG3-c2 (BG3) cells were cultured and proteins were depleted by RNAi as described (Schaaf et al. 2009). Cells were treated with 
$10 \mu \mathrm{M}$ JQ1 in the medium for $8 \mathrm{~h}$ to inhibit Fs(1)h binding, and cell cycle stages were determined by propidium iodide staining and FACS analysis in the Saint Louis University Flow Core.

\section{ChIP-seq and quantification}

ChIP-seq was performed and quantified as detailed elsewhere (Dorsett and Misulovin 2017) using concurrent experiments, overlapping sets of chromatin preparations, multiple biological repeats, and validated antibodies (Supplemental Methods; Supplemental Table S1).

Enhancers, PREs, and active promoters were identified and defined as 500-bp sequences based on DNase I hypersensitivity, histone modifications (H3K4me1, H3K4me3, H3K27me3, H3K27ac), and PRO-seq data (Schaaf et al. 2013a,b; Swain et al. 2016; Pherson et al. 2017; Misulovin et al. 2018). Occupancy of individual features was calculated using BED files and scripts provided in prior publications (Swain et al. 2016; Pherson et al. 2017; Misulovin et al. 2018). Details are in Supplemental Methods.

\section{Early S phase DNA replication}

Early $\mathrm{S}$ phase DNA replication was quantified by adapting an origin-mapping protocol (MacAlpine et al. 2004) with EdU thymidine analog detection of newly synthesized DNA (Ramachandran and Henikoff 2016). Details are in Supplemental Methods.

\section{MEDI and MED30 antibodies}

A His(6) fusion to the 1140-1475 C terminal residues of MED1 was expressed in $E$. coli, purified by nickel chromatography under denaturing conditions, and insoluble protein was used to immunize a rabbit at Josman, LLC. A His(6) fusion to full-length MED30 (residues 1-318) was expressed in E. coli, purified by nickel chromatography, and the insoluble protein used to immunize a guinea pig at Pocono Rabbit Farm and Laboratory. Antibody spec-

\begin{tabular}{|c|c|c|c|c|}
\hline$A_{\text {genotype }}$ & $f s(1) h^{1} Y$ & q $f \boldsymbol{s}(1) h^{1} /+$ & $\%$ expected & P vs. $+/+$ \\
\hline$+/+$ & 67 & 108 & 62 & \\
\hline Nipped $-B^{407} /+$ & 7 & 154 & 5 & 2.7E-15 \\
\hline Nipped-B $B^{N C 41 /+}$ & 21 & 85 & 25 & $7.9 \mathrm{E}-4$ \\
\hline$v t d^{4} /+$ & 8 & 66 & 12 & $5.3 \mathrm{E}-6$ \\
\hline$v t d^{e \times 15} /+$ & 21 & 43 & 49 & 2.7E-1 \\
\hline
\end{tabular}

B

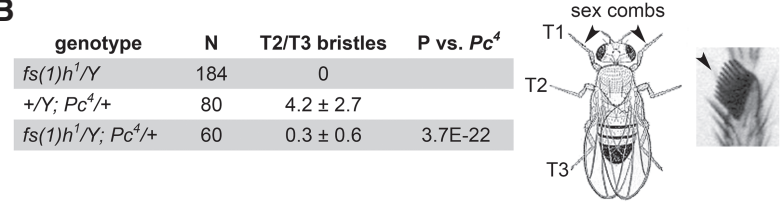

\begin{tabular}{|c|c|c|c|c|}
\hline genotype & $\mathbf{N}$ & T1 bristles & P vs. $f s(1) h^{1}$ & P vs. Nipped-B ${ }^{407}$ \\
\hline$f s(1) h^{1} / Y$ & 30 & $11.1 \pm 0.9$ & & \\
\hline$+/ Y ;$ Nipped- $B^{407 /+}$ & 30 & $10.7 \pm 0.9$ & $3.8 \mathrm{E}-2$ & \\
\hline$f_{S}(1) h^{1} / Y ;$ Nipped- $B^{407} /+$ & 8 & $8.9 \pm 0.4$ & $4.4 \mathrm{E}-8$ & $7.8 \mathrm{E}-6$ \\
\hline
\end{tabular}

Figure 9. Nipped-B and Rad21 ( $v t d$ ) mutations dominantly enhance $f_{s}(1) h^{\top}$ mutant phenotypes. Crosses were conducted at $29^{\circ}$. (A) Nipped-B and $\operatorname{Rad} 21$ ( $v t d$ ) mutations dominantly decrease $f_{s}(1) h^{1}$ male viability. The numbers of $f_{s}(1) h^{1} / Y$ males with the indicated genotypes and their $f_{s}(1) h^{1} /+$ sisters recovered are given. The percentage expected is the male to female ratio. $P$-values were calculated using Fisher's exact test. (B) $f s(1) h^{1}$ suppresses the ectopic T2 and T3 leg sex comb bristles in $P c^{4}$ mutant males and reduces the number of $\mathrm{T} 1$ sex comb bristles when combined with heterozygous Nipped- $-B^{407}$. The diagram shows a male fly indicating the $\mathrm{T} 1, \mathrm{~T} 2$, and $\mathrm{T} 3$ legs and a magnified view of $\mathrm{T} 1$ sex comb bristles. The tables give the number of legs scored $(\mathrm{N})$ with the average number of bristles per leg and the standard deviation. $P$-values were calculated using the $t$-test.

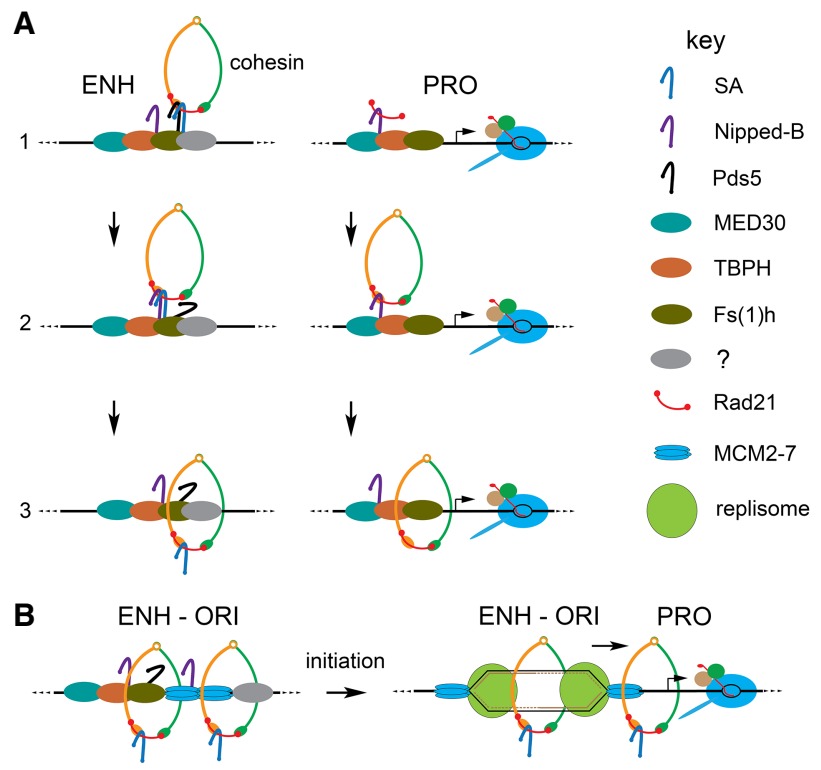

Figure 10. Theoretical models for cohesin recruitment and the roles of origins and DNA replication in sister chromatid cohesion and enhancerpromoter communication. $(A)$ Cohesin recruitment to enhancers (ENH) and promoters (PRO). The factor key is on the right. At enhancers (left), we posit that Pds5 (black) and SA (blue) recruit tripartite cohesin rings (step 1) and that Nipped-B (purple) displaces Pds5 (step 2) to load cohesin topologically (step 3). SA association with enhancers is facilitated by Pds5 (Misulovin et al. 2018) and unknown enhancer-specific proteins (gray). At promoters (right), we envision that the MED30 Mediator subunit (cyan) and the TBPH protein (orange) (Swain et al. 2016) recruit Nipped-B and Rad21 (red) without SMC1-SMC3 dimers or SA (step 1). At some frequency, Nipped-B-Rad21 complexes capture SMC1-SMC3 dimers (step 2) and SA-deficient cohesin is loaded (step 3). (B) We theorize that enhancers capture translocating MCM2-7 helicase complexes (Powell et al. 2015) to position early replication origins (left). DNA unwinding by MCM2-7 upon initiation of replication topologically captures both single-stranded templates within cohesin rings behind the nascent forks to establish sister chromatid cohesion (right). Replication forks push other SA-containing cohesin rings to be captured by neighboring promoters, facilitating enhancer-promoter communication (right).

ificities were confirmed by western blots of whole-cell extracts of control and RNAi-depleted BG3 cells (Supplemental Fig. S3A).

\section{Genetic crosses}

Drosophila stocks were maintained and crosses conducted as described (Rollins et al. 1999). $f s(1) h^{1}$ stocks were obtained from the Bloomington Drosophila Stock Center at Indiana University. Nipped-B, cohesin, and Pc mutant stocks were described previously (Rollins et al. 1999; Gause et al. 2008; Schaaf et al. 2013b). Fisher's exact test and $t$-tests were used as indicated in Figure 9.

\section{Data access}

ChIP-seq data from this study have been submitted to the NCBI Gene Expression Omnibus (GEO; https://www.ncbi.nlm.nih .gov/geo/) under accession number GSE118484.

\section{Acknowledgments}

We thank Igor Dawid (NICHD) for Fs(1)h antibodies, Fran Sverdrup (Saint Louis University) for JQ1, and Srinivas Ramachandran (University of Colorado) for helpful discussions 
about the EdU experiments. We thank Frank Uhlmann (Francis Crick Institute) and Hongtao Yu (HHMI, UT Southwestern) for helpful comments on the manuscript. This work was supported by grants from the National Institutes of Health (R01 GM108714) to D.D. and the Cornelia de Lange Syndrome Foundation to M.P.

\section{References}

Allen BL, Taatjes DJ. 2015. The Mediator complex: a central integrator of transcription. Nat Rev Mol Cell Biol 16: 155-166. doi:10.1038/nrm3951

Beckouët F, Srinivasan M, Roig MB, Chan KL, Scheinost JC, Batty P, Hu B, Petela N, Gligoris T, Smith AC, et al. 2016. Releasing activity disengages cohesin's Smc3/Scc1 interface in a process blocked by acetylation. Mol Cell 61: 563-574. doi:10.1016/j.molcel.2016.01.026

Brough R, Bajrami I, Vatcheva R, Natrajan R, Reis-Filho JS, Lord CJ, Ashworth A. 2012. APRIN is a cell cycle specific BRCA2-interacting protein required for genome integrity and a predictor of outcome after chemotherapy in breast cancer. EMBO J 31: 1160-1176. doi:10.1038/emboj .2011.490

Camdere G, Guacci V, Stricklin J, Koshland D. 2015. The ATPases of cohesin interface with regulators to modulate cohesin-mediated DNA tethering. eLife 4: e11315. doi:10.7554/eLife.11315

Countryman P, Fan Y, Gorthi A, Pan H, Strickland J, Kaur P, Wang X, Lin J, Lei X, White C, et al. 2018. Cohesin SA2 is a sequence-independent DNA-binding protein that recognizes DNA replication and repair intermediates. J Biol Chem 293: 1054-1069. doi:10.1074/jbc.M117.806406

Dey A, Ellenberg J, Farina A, Coleman AE, Maruyama T, Sciortino S, Lippincott-Schwartz J, Ozato K. 2000. A bromodomain protein, MCAP, associates with mitotic chromosomes and affects $\mathrm{G}_{2}$-to-M transition. Mol Cell Biol 20: 6537-6549. doi:10.1128/MCB.20.17.6537-6549 .2000

Digan ME, Haynes SR, Mozer BA, Dawid IB, Forquignon F, Gans M. 1986. Genetic and molecular analysis of $f s(1) h$, a maternal effect homeotic gene in Drosophila. Dev Biol 114: 161-169. doi:10.1016/0012-1606 (86)90392-1

Dorsett D, Merkenschlager M. 2013. Cohesin at active genes: a unifying theme for cohesin and gene expression from model organisms to humans. Curr Opin Cell Biol 25: 327-333. doi:10.1016/j.ceb.2013.02.003

Dorsett D, Misulovin Z. 2017. Measuring sister chromatid cohesion protein genome occupancy in Drosophila melanogaster by ChIP-seq. Methods Mol Biol 1515: 125-139. doi:10.1007/978-1-4939-6545-8 8

Dorsett D, Ström L. 2012. The ancient and evolving roles of cohesin in gene expression and DNA repair. Curr Biol 22: R240-R250. doi:10.1016/j.cub .2012 .02 .046

Eaton ML, Prinz JA, MacAlpine HK, Tretyakov G, Kharchenko PV, MacAlpine DM. 2011. Chromatin signatures of the Drosophila replication program. Genome Res 21: 164-174. doi:10.1101/gr.116038.110

Elbatsh AMO, Haarhuis JHI, Petela N, Chapard C, Fish A, Celie PH, Stadnik M, Ristic D, Wyman C, Medema RH, et al. 2016. Cohesin releases DNA through asymmetric ATPase-driven ring opening. Mol Cell 61: 575-588. doi:10.1016/j.molcel.2016.01.025

Filippakopoulos P, Qi J, Picaud S, Shen Y, Smith WB, Fedorov O, Morse EM, Keates T, Hickman TT, Felletar I, et al. 2010. Selective inhibition of BET bromodomains. Nature 468: 1067-1073. doi:10.1038/nature09504

Florence BL, Faller DV. 2008. Drosophila female sterile (1) homeotic is a multifunctional transcriptional regulator that is modulated by Ras signaling. Dev Dyn 237: 554-564. doi:10.1002/dvdy.21432

Gans M, Audit C, Masson M. 1975. Isolation and characterization of sexlinked female-sterile mutants in Drosophila melanogaster. Genetics 81: 683-704.

Gause M, Webber HA, Misulovin Z, Haller G, Rollins RA, Eissenberg JC, Bickel SE, Dorsett D. 2008. Functional links between Drosophila Nipped-B and cohesin in somatic and meiotic cells. Chromosoma 117: 51-66. doi:10.1007/s00412-007-0125-5

Gause M, Misulovin Z, Bilyeu A, Dorsett D. 2010. Dosage-sensitive regulation of cohesin chromosome binding and dynamics by Nipped-B, Pds5, and Wapl. Mol Cell Biol 30: 4940-4951. doi:10.1128/MCB .00642-10

Gillespie PJ, Hirano T. 2004. Scc2 couples replication licensing to sister chromatid cohesion in Xenopus egg extracts. Curr Biol 14: 1598-1603. doi:10 .1016/j.cub.2004.07.053

Guruharsha KG, Obar RA, Mintseris J, Aishwarya K, Krishnan RT, Vijayraghavan K, Artavanis-Tsakonas S. 2012. Drosophila protein interaction map (DPiM): a paradigm for metazoan protein complex interactions. Fly (Austin) 6: 246-253. doi:10.4161/fly.22108

Hallson G, Syrzycka M, Beck SA, Kennison JA, Dorsett D, Page SL, Hunter SM, Keall R, Warren WD, Brock HW, et al. 2008. The Drosophila cohesin subunit Rad21 is a trithorax group (trxG) protein. Proc Natl Acad Sci 105: 12405-12410. doi:10.1073/pnas.0801698105

Hsu SC, Blobel GA. 2017. The role of bromodomain and extraterminal motif (BET) proteins in chromatin structure. Cold Spring Harb Symp Quant Biol 82: 37-43. doi:10.1101/sqb.2017.82.033829

Kagey MH, Newman JJ, Bilodeau S, Zhan Y, Orlando DA, van Berkum NL Ebmeier CC, Goossens J, Rahl PB, Levine SS, et al. 2010. Mediator and cohesin connect gene expression and chromatin architecture. Nature 467: 430-435. doi:10.1038/nature09380

Kanke M, Tahara E, Huis In't Veld PJ, Nishiyama T. 2016. Cohesin acetylation and Wapl-Pds5 oppositely regulate translocation of cohesin along DNA. EMBO J 35: 2686-2698. doi:10.15252/embj.201695756

Kennison JA, Tamkun JW. 1988. Dosage-dependent modifiers of polycomb and antennapedia mutations in Drosophila. Proc Natl Acad Sci 85: 81368140. doi:10.1073/pnas.85.21.8136

Kojic A, Cuadrado A, De Koninck M, Giménez-Llorente D, RodríguezCorsino M, Gómez-López G, Le Dily F, Marti-Renom MA, Losada A 2018. Distinct roles of cohesin-SA1 and cohesin-SA2 in 3D chromosome organization. Nat Struct Mol Biol 25: 496-504. doi:10.1038/s41594-0180070-4

Kulemzina I, Schumacher MR, Verma V, Reiter J, Metzler J, Failla AV, Lanz C, Sreedharan VT, Rätsch G, Ivanov D. 2012. Cohesin rings devoid of Scc3 and Pds5 maintain their stable association with the DNA. PLoS Genet 8: e1002856. doi:10.1371/journal.pgen.1002856

Kusch T. 2015. Brca2-Pds5 complexes mobilize persistent meiotic recombination sites to the nuclear envelope. J Cell Sci 128: 717-727. doi:10 $.1242 /$ jcs. 159988

Laugsch M, Seebach J, Schnittler H, Jessberger R. 2013. Imbalance of SMC1 and SMC3 cohesins causes specific and distinct effects. PLoS One 8: e65149. doi:10.1371/journal.pone.0065149

MacAlpine DM, Rodríguez HK, Bell SP. 2004. Coordination of replication and transcription along a Drosophila chromosome. Genes Dev 18: 3094-3105. doi:10.1101/gad.1246404

MacAlpine HK, Gordân R, Powell SK, Hartemink AJ, MacAlpine DM. 2010. Drosophila ORC localizes to open chromatin and marks sites of cohesin complex loading. Genome Res 20: 201-211. doi:10.1101/gr.097873.109

Misulovin Z, Schwartz YB, Li XY, Kahn TG, Gause M, MacArthur S, Fay JC, Eisen MB, Pirrotta V, Biggin MD, et al. 2008. Association of cohesin and Nipped-B with transcriptionally active regions of the Drosophila melanogaster genome. Chromosoma 117: 89-102. doi:10.1007/s00412-0070129-1

Misulovin Z, Pherson M, Gause M, Dorsett D. 2018. Brca2, Pds5 and Wap differentially control cohesin chromosome association and function. PLoS Genet 14: e1007225. doi:10.1371/journal.pgen.1007225

Morales C, Losada A. 2018. Establishing and dissolving cohesion during the vertebrate cell cycle. Curr Opin Cell Biol 52: 51-57. doi:10.1016/j.ceb .2018.01.010

Mullegama SV, Klein SD, Mulatinho MV, Senaratne TN, Singh K; UCLA Clinical Genomics Center, Nguyen DC, Gallant NM, Strom SP, Ghahremani S, et al. 2017. De novo loss-of-function variants in STAG2 are associated with developmental delay, microcephaly, and congenital anomalies. Am J Med Genet A 173: 1319-1327. doi:10 1002/ajmg.a.38207

Mullegama SV, Klein SD, Signer RH; UCLA Clinical Genomics Center, Vilain E, Martinez-Agosto JA. 2019. Mutations in STAG2 cause an X-linked cohesinopathy associated with undergrowth, developmental delay, and dysmorphia: expanding the phenotype in males. Mol Genet Genomic Med 7: e501. doi:10.1002/mgg3.501

Murayama Y, Uhlmann F. 2014. Biochemical reconstitution of topological DNA binding by the cohesin ring. Nature 505: 367-371. doi:10.1038/ nature 12867

Murayama Y, Uhlmann F. 2015. DNA entry into and exit out of the cohesin ring by an interlocking gate mechanism. Cell 163: 1628-1640. doi:10 1016/j.cell.2015.11.030

Neuwald AF, Hirano T. 2000. HEAT repeats associated with condensins, cohesins, and other complexes involved in chromosome-related functions. Genome Res 10: 1445-1452. doi:10.1101/gr.147400

Olley G, Ansari M, Bengani H, Grimes GR, Rhodes J, von Kriegsheim A, Blatnik A, Stewart FJ, Wakeling E, Carroll N, et al. 2018. BRD4 interacts with NIPBL and BRD4 is mutated in a Cornelia de Lange-like syndrome. Nat Genet 50: 329-332. doi:10.1038/s41588-018-0042-y

Ouyang Z, Yu H. 2017. Releasing the cohesin ring: a rigid scaffold model for opening the DNA exit gate by Pds5 and Wapl. Bioessays 39: 1600207. doi:10.1002/bies.201600207

Pauli A, Althoff F, Oliveira RA, Heidmann S, Schuldiner O, Lehner CF, Dickson BJ, Nasmyth K. 2008. Cell-type-specific TEV protease cleavage reveals cohesin functions in Drosophila neurons. Dev Cell 14: 239251. doi:10.1016/j.devcel.2007.12.009

Petela NJ, Gligoris TG, Metson J, Lee BG, Voulgaris M, Hu B, Kikuchi S, Chapard C, Chen W, Rajendra E, et al. 2018. Scc2 is a potent activator 
of cohesin's ATPase that promotes loading by binding Scc1 without Pds5. Mol Cell 70: 1134-1148.e7. doi:10.1016/j.molcel.2018.05.022

Pherson M, Misulovin Z, Gause M, Mihindukulasuriya K, Swain A, Dorset D. 2017. Polycomb repressive complex 1 modifies transcription of active genes. Sci Adv 3: e1700944. doi:10.1126/sciadv.1700944

Powell SK, MacAlpine HK, Prinz JA, Li Y, Belsky JA, MacAlpine DM. 2015 Dynamic loading and redistribution of the Mcm2-7 helicase complex through the cell cycle. EMBO J 34: 531-543. doi:10.15252/embj .201488307

Ramachandran S, Henikoff S. 2016. Transcriptional regulators compete with nucleosomes post-replication. Cell 165: 580-592. doi:10.1016/j cell.2016.02.062

Rhodes JDP, Haarhuis JHI, Grimm JB, Rowland BD, Lavis LD, Nasmyth KA 2017. Cohesin can remain associated with chromosomes during DNA replication. Cell Rep 20: 2749-2755. doi:10.1016/j.celrep.2017.08.092

Rollins RA, Morcillo P, Dorsett D. 1999. Nipped-B, a Drosophila homologue of chromosomal adherins, participates in activation by remote enhancers in the cut and Ultrabithorax genes. Genetics 152: 577-593.

Schaaf CA, Misulovin Z, Sahota G, Siddiqui AM, Schwartz YB, Kahn TG, Pirrotta V, Gause M, Dorsett D. 2009. Regulation of the Drosophila Enhancer of split and invected-engrailed gene complexes by sister chromatid cohesion proteins. PLoS One 4: e6202. doi:10.1371/journal.pone .0006202

Schaaf CA, Kwak H, Koenig A, Misulovin Z, Gohara DW, Watson A, Zhou Y, Lis JT, Dorsett D. 2013a. Genome-wide control of RNA polymerase II activity by cohesin. PLoS Genet 9: e1003382. doi:10.1371/journal.pgen 1003382

Schaaf CA, Misulovin Z, Gause M, Koenig A, Gohara DW, Watson A, Dorsett D. 2013b. Cohesin and Polycomb proteins functionally interact to control transcription at silenced and active genes. PLoS Genet 9: e1003560. doi:10.1371/journal.pgen.1003560

Skibbens RV. 2009. Establishment of sister chromatid cohesion. Curr Bio 19: R1126-R1132. doi:10.1016/j.cub.2009.10.067

Soardi FC, Machado-Silva A, Linhares ND, Zheng G, Qu Q, Pena HB, Martins TMM, Vieira HGS, Pereira NB, Melo-Minardi RC, et al. 2017. Familial STAG2 germline mutation defines a new human cohesinopathy. NP Genom Med 2: 7. doi:10.1038/s41525-017-0009-4

Swain A, Misulovin Z, Pherson M, Gause M, Mihindukulasuriya K, Rickels RA, Shilatifard A, Dorsett D. 2016. Drosophila TDP-43 RNA-binding pro- tein facilitates association of sister chromatid cohesion proteins with genes, enhancers and Polycomb response elements. PLoS Genet 12: e1006331. doi:10.1371/journal.pgen.1006331

Takahashi TS, Yiu P, Chou MF, Gygi S, Walter JC. 2004. Recruitment of Xenopus Scc 2 and cohesin to chromatin requires the pre-replication complex. Nat Cell Biol 6: 991-996. doi:10.1038/ncb1177

Takahashi TS, Basu A, Bermudez V, Hurwitz J, Walter JC. 2008. Cdc7-Drf1 kinase links chromosome cohesion to the initiation of DNA replication in Xenopus egg extracts. Genes Dev 22: 1894-1905. doi:10.1101/gad .1683308

Uhlmann F. 2016. SMC complexes: from DNA to chromosomes. Nat Rev Mol Cell Biol 17: 399-412. doi:10.1038/nrm.2016.30

Villa-Hernández S, Bermejo R. 2018. Cohesin dynamic association to chromatin and interfacing with replication forks in genome integrity maintenance. Curr Genet 64: 1005-1013. doi:10.1007/s00294-018-0824-x

Wells JN, Gligoris TG, Nasmyth KA, Marsh JA. 2017. Evolution of condensin and cohesin complexes driven by replacement of Kite by Hawk proteins. Curr Biol 27: R17-R18. doi:10.1016/j.cub.2016.11.050

Wu Y, Gause M, Xu D, Misulovin Z, Schaaf CA, Mosarla RC, Mannino E, Shannon M, Jones E, Shi M, et al. 2015. Drosophila Nipped-B mutants model Cornelia de Lange Syndrome in growth and behavior. PLoS Genet 11: e1005655. doi:10.1371/journal.pgen.1005655

Yu H. 2016. Magic acts with the cohesin ring. Mol Cell 61: 489-491. doi:10 .1016/j.molcel.2016.02.003

Yuan B, Neira J, Pehlivan D, Santiago-Sim T, Song X, Rosenfeld J, Posey JE, Patel V, Jin W, Adam MP, et al. 2018. Clinical exome sequencing reveals locus heterogeneity and phenotypic variability of cohesinopathies. Genet Med 30. doi:10.1038/s41436-018-0085-6

Zhao R, Nakamura T, Fu Y, Lazar Z, Spector DL. 2011. Gene bookmarking accelerates the kinetics of post-mitotic transcriptional re-activation. Nat Cell Biol 13: 1295-1304. doi:10.1038/ncb2341

Zheng G, Kanchwala M, Xing C, Yu H. 2018. MCM2-7-dependent cohesin loading during $\mathrm{S}$ phase promotes sister-chromatid cohesion. eLife 7: e33920. doi:10.7554/eLife.33920

Received September 7, 2018; accepted in revised form February 20, 2019.

\section{Genome Research}

www.genome.org 


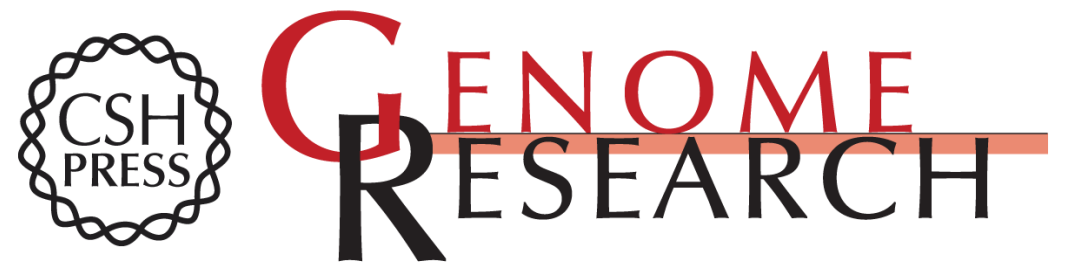

\section{Cohesin occupancy and composition at enhancers and promoters are linked to DNA replication origin proximity in Drosophila}

Michelle Pherson, Ziva Misulovin, Maria Gause, et al.

Genome Res. 2019 29: 602-612 originally published online February 22, 2019

Access the most recent version at doi:10.1101/gr.243832.118

Supplemental Material

References

Open Access

Creative Commons

License

Email Alerting Service
http://genome.cshlp.org/content/suppl/2019/03/18/gr.243832.118.DC1

This article cites 63 articles, 15 of which can be accessed free at: http://genome.cshlp.org/content/29/4/602.full.html\#ref-list-1

Freely available online through the Genome Research Open Access option.

This article, published in Genome Research, is available under a Creative Commons License (Attribution 4.0 International), as described at http://creativecommons.org/licenses/by/4.0/.

Receive free email alerts when new articles cite this article - sign up in the box at the top right corner of the article or click here.

\section{Affordable, Accurate Sequencing.}

